\title{
Tau Physics: Theory Overview
}

\author{
A. Pich ${ }^{\mathrm{a}}$ \\ åDepartament de Física Teòrica, IFIC, Univ. València-CSIC, Apt. 22085, E-46071 València, Spain
}

The present status of some selected topics on $\tau$ physics is presented: charged-current universality tests, bounds on lepton-flavour violation, the determination of $\alpha_{s}$ from the inclusive $\tau$ hadronic width, the measurement of $\left|V_{u s}\right|$ through the Cabibbo-suppressed decays of the $\tau$, and the theoretical description of the $\tau \rightarrow \nu_{\tau} K \pi$ spectrum.

\section{Introduction}

The known leptons provide clean probes to precisely test the Standard Model and search for signals of new dynamics. The electroweak gauge structure has been successfully tested at the $0.1 \%$ to $1 \%$ level, confirming the Standard Model framework [1]. Moreover, the hadronic $\tau$ decays turn out to be a beautiful laboratory for studying strong interaction effects at low energies [2]. The $\tau$ is the only known lepton massive enough to decay into hadrons. Its semileptonic decays are then ideally suited for studying the hadronic weak currents. Accurate determinations of the QCD coupling, $\left|V_{u s}\right|$ and the strange quark mass have been obtained with $\tau$ decay data.

The huge statistics accumulated at the B Factories allow to explore lepton-flavour-violating $\tau$ decay modes with increased sensitivities beyond $10^{-7}$, which could be further pushed down to few $10^{-9}$ at future facilities. Moreover, BESIII will soon start taking data at threshold, providing complementary information on the $\tau$, such as an improved mass measurement. Thus, $\tau$ physics is entering a new era, full of interesting possibilities and with a high potential for new discoveries.

\section{Tests on charged-current universality}

In the Standard Model all lepton doublets have identical couplings to the $W$ boson. Comparing the measured decay widths of leptonic or semileptonic decays which only differ in the lepton flavour, one can test experimentally that the $W$ interaction is indeed the same, i.e. that
Table 1

Present constraints on $\left|g_{l} / g_{l^{\prime}}\right|$.

\begin{tabular}{lc}
\hline & $\left|g_{\mu} / g_{e}\right|$ \\
\hline$B_{\tau \rightarrow \mu} / B_{\tau \rightarrow e}$ & $1.0000 \pm 0.0020$ \\
$B_{\pi \rightarrow \mu} / B_{\pi \rightarrow e}$ & $1.0021 \pm 0.0016$ \\
$B_{K \rightarrow \mu} / B_{K \rightarrow e}$ & $1.004 \pm 0.007$ \\
$B_{K \rightarrow \pi \mu} / B_{K \rightarrow \pi e}$ & $1.002 \pm 0.002$ \\
$B_{W \rightarrow \mu} / B_{W \rightarrow e}$ & $0.997 \pm 0.010$ \\
\hline \hline & $\left|g_{\tau} / g_{\mu}\right|$ \\
\hline$B_{\tau \rightarrow e} \tau_{\mu} / \tau_{\tau}$ & $1.0006 \pm 0.0022$ \\
$\Gamma_{\tau \rightarrow \pi} / \Gamma_{\pi \rightarrow \mu}$ & $0.996 \pm 0.005$ \\
$\Gamma_{\tau \rightarrow K} / \Gamma_{K \rightarrow \mu}$ & $0.979 \pm 0.017$ \\
$B_{W \rightarrow \tau} / B_{W \rightarrow \mu}$ & $1.039 \pm 0.013$ \\
\hline \hline & $\left|g_{\tau} / g_{e}\right|$ \\
\hline$B_{\tau \rightarrow \mu} \tau_{\mu} / \tau_{\tau}$ & $1.0005 \pm 0.0023$ \\
$B_{W \rightarrow \tau} / B_{W \rightarrow e}$ & $1.036 \pm 0.014$ \\
\hline
\end{tabular}

$g_{e}=g_{\mu}=g_{\tau} \equiv g$. As shown in Table 1, the present data verify the universality of the leptonic charged-current couplings to the $0.2 \%$ level.

The $\tau$ leptonic branching fractions and the $\tau$ lifetime are known with a precision of $0.3 \%$ [2]. A slightly improved lifetime measurement could be expected from BaBar and Belle [3]. For comparison, the $\mu$ lifetime is already known with an accuracy of $10^{-5}$, which should be further improved to $10^{-6}$ by the MuLan [4] and FAST [5] experiments at PSI. The universality tests require also a good determination of $m_{\tau}^{5}$, which is only known to the $0.06 \%$ level [6]. Two new measurements of 
Table 2

Best published limits (90\% CL) on lepton-flavour-violating decays [6, 24, 25].

\begin{tabular}{lcccllllll}
\hline $\operatorname{Br}\left(\mu^{-} \rightarrow \mathrm{X}^{-}\right) \cdot 10^{12}$ & & & & & & & \\
$e^{-} \gamma$ & 12 & $e^{-} 2 \gamma$ & 72 & $e^{-} e^{-} e^{+}$ & 1.0 & & & \\
\hline \hline $\operatorname{Br}\left(\tau^{-} \rightarrow \mathrm{X}^{-}\right) \cdot 10^{8}$ & & & & & & & \\
$e^{-} \gamma$ & 11 & $e^{-} e^{+} e^{-}$ & 3.6 & $e^{-} \mu^{+} \mu^{-}$ & 3.7 & $e^{-} e^{-} \mu^{+}$ & 2.0 & $e^{-} \pi^{0}$ & 8.0 \\
$\mu^{-} \gamma$ & 4.5 & $\mu^{-} e^{+} e^{-}$ & 2.7 & $\mu^{-} \mu^{+} \mu^{-}$ & 3.2 & $\mu^{-} \mu^{-} e^{+}$ & 2.3 & $\mu^{-} \pi^{0}$ & 11 \\
$e^{-} \eta$ & 9.2 & $e^{-} \eta^{\prime}$ & 16 & $e^{-} \rho^{0}$ & 6.3 & $e^{-} \omega$ & 11 & $e^{-} \phi$ & 7.3 \\
$\mu^{-} \eta$ & 6.5 & $\mu^{-} \eta^{\prime}$ & 13 & $\mu^{-} \rho^{0}$ & 6.8 & $\mu^{-} \omega$ & 8.9 & $\mu^{-} \phi$ & 13 \\
$e^{-} K_{S}$ & 5.6 & $e^{-} K^{* 0}$ & 7.8 & $e^{-} \bar{K}^{* 0}$ & 7.7 & $e^{-} K^{+} \pi^{-}$ & 16 & $e^{-} \pi^{+} K^{-}$ & 32 \\
$\mu^{-} K_{S}$ & 4.9 & $\mu^{-} K^{* 0}$ & 5.9 & $\mu^{-} \bar{K}^{* 0}$ & 10 & $\mu^{-} K^{+} \pi^{-}$ & 32 & $\mu^{-} \pi^{+} K^{-}$ & 26 \\
$e^{-} K^{+} K^{-}$ & 14 & $e^{-} \pi^{+} \pi^{-}$ & 12 & $e^{+} \pi^{-} \pi^{-}$ & 20 & $e^{+} K^{-} K^{-}$ & 15 & $e^{+} \pi^{-} K^{-}$ & 18 \\
$\mu^{-} K^{+} K^{-}$ & 25 & $\mu^{-} \pi^{+} \pi^{-}$ & 29 & $\mu^{+} \pi^{-} \pi^{-}$ & 7 & $\mu^{+} K^{-} K^{-}$ & 44 & $\mu^{+} \pi^{-} K^{-}$ & 22 \\
$\bar{\Lambda} \pi^{-}$ & 14 & $\Lambda \pi^{-}$ & 7.2 & & & & & & \\
\hline
\end{tabular}

the $\tau$ mass have been published recently:

$m_{\tau}=\left\{\begin{array}{lr}1776.61 \pm 0.13 \pm 0.35 \mathrm{MeV} & {[\text { Belle }]} \\ 1776.81_{-0.23}^{+0.25} \pm 0.15 \mathrm{MeV} & {[\text { KEDR }]}\end{array}\right.$

Belle [7] has made a pseudomass analysis of $\tau \rightarrow$ $\nu_{\tau} 3 \pi$ decays, while KEDR [8] measures the $\tau^{+} \tau^{-}$ threshold production, taking advantage of a precise energy calibration through the resonance depolarization method. In both cases the achieved precision is getting close to the previous BESdominated value, $m_{\tau}=1776.99_{-0.26}^{+0.29}$ [6]. KEDR aims to obtain a final accuracy of $0.15 \mathrm{MeV}$. A precision better than $0.05 \mathrm{MeV}$ should be easily achieved at BESIII [9], through a detailed analysis of $\sigma\left(e^{+} e^{-} \rightarrow \tau^{+} \tau^{-}\right)$at threshold [10-12].

Table[1 shows also the contraints obtained from pion [6] and kaon decays [13], applying the recently calculated radiative corrections at NLO in chiral perturbation theory $[14,15]$. The accuracy achieved with $K_{l 3}$ data is already comparable to the one obtained from $\tau$ or $\pi_{l 2}$ decays.

Owing to the limited statistics available, the decays $W^{-} \rightarrow l^{-} \nu_{l}$ only test universality at the $1 \%$ level. At present, $\operatorname{Br}\left(W \rightarrow \nu_{\tau} \tau\right)$ is $2.1 \sigma / 2.7 \sigma$ larger than $\operatorname{Br}\left(W \rightarrow \nu_{e} e / \nu_{\mu} \mu\right)$ [16]. The stringent limits on $\left|g_{\tau} / g_{e, \mu}\right|$ from $W$-mediated decays make unlikely that this is a real effect.

\section{Lepton-flavour violating decays}

We have now clear experimental evidence that neutrinos are massive particles and there is mixing in the lepton sector. The smallness of neutrino masses implies a strong suppression of neutrinoless lepton-flavour-violating processes, which can be avoided in models with sources of lepton flavour violation not related to $m_{\nu_{i}}$. The scale of the flavour-violating new-physics interactions can be constrained imposing the requirement of a viable leptogenesis. Recent studies within different new-physics scenarios find interesting correlations between $\mu$ and $\tau$ lepton-flavour-violating decays, with $\mu \rightarrow e \gamma$ often expected to be close to the present exclusion limit [17-23].

The B Factories are pushing the experimental limits on neutrinoless lepton-flavour-violating $\tau$ decays beyond the $10^{-7}$ level $[24,25]$, increasing in a drastic way the sensitivity to new physics scales. Future experiments could push further some limits to the $10^{-9}$ level [26], allowing to explore interesting and totally unknown phenomena. Complementary information will be provided by the MEG experiment, which will search for $\mu^{+} \rightarrow e^{+} \gamma$ events with a sensitivity of $10^{-13}$ [27]. There are also ongoing projects at J-PARC aiming to study $\mu \rightarrow e$ conversions in muonic atoms, at the $10^{-16}[28]$ or even $10^{-18}$ [29] level. 


\section{The inclusive hadronic width of the tau}

The inclusive character of the total $\tau$ hadronic width renders possible an accurate calculation of the ratio $[30-34]$

$R_{\tau} \equiv \frac{\Gamma\left[\tau^{-} \rightarrow \nu_{\tau} \text { hadrons }\right]}{\Gamma\left[\tau^{-} \rightarrow \nu_{\tau} e^{-} \bar{\nu}_{e}\right]}=R_{\tau, V}+R_{\tau, A}+R_{\tau, S}$.

Using analyticity constraints and the Operator Product Expansion, one can separately compute the contributions associated with specific quark currents: $R_{\tau, V}$ and $R_{\tau, A}$ correspond to the Cabibbo-allowed decays through the vector and axial-vector currents, while $R_{\tau, S}$ contains the remaining Cabibbo-suppressed contributions.

The combination $R_{\tau, V+A}$ can be written as [32]

$R_{\tau, V+A}=N_{C}\left|V_{u d}\right|^{2} S_{\mathrm{EW}}\left\{1+\delta_{\mathrm{P}}+\delta_{\mathrm{NP}}\right\}$,

where $N_{C}=3$ is the number of quark colours and $S_{\mathrm{EW}}=1.0201 \pm 0.0003$ contains the electroweak radiative corrections [35-37]. The dominant correction $(\sim 20 \%)$ is the perturbative QCD contribution $\delta_{\mathrm{P}}$, which is already known to $O\left(\alpha_{s}^{4}\right)$ $[32,38]$ and includes a resummation of the most important higher-order effects [33,39].

Non-perturbative contributions are suppressed by six powers of the $\tau$ mass [32] and, therefore, are very small. Their numerical size has been determined from the invariant-mass distribution of the final hadrons in $\tau$ decay, through the study of weighted integrals [40],

$R_{\tau}^{k l} \equiv \int_{0}^{m_{\tau}^{2}} d s\left(1-\frac{s}{m_{\tau}^{2}}\right)^{k}\left(\frac{s}{m_{\tau}^{2}}\right)^{l} \frac{d R_{\tau}}{d s}$,

which can be calculated theoretically in the same way as $R_{\tau}$. The predicted suppression [32] of the non-perturbative corrections has been confirmed by ALEPH [41], CLEO [42] and OPAL [43]. The most recent analysis [44] gives

$\delta_{\mathrm{NP}}=0.0001 \pm 0.0017$.

The QCD prediction for $R_{\tau, V+A}$ is then completely dominated by $\delta_{P}$; non-perturbative effects being smaller than the perturbative uncertainties from uncalculated higher-order corrections. The result turns out to be very sensitive to the value of $\alpha_{s}\left(m_{\tau}\right)$, allowing for an accurate determination of

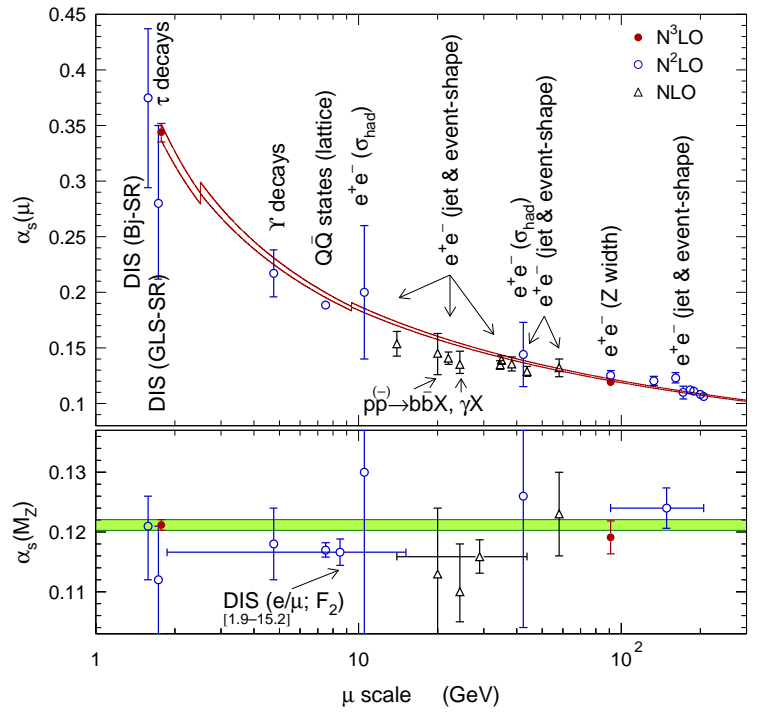

Figure 1. Measured values of $\alpha_{s}$ at different scales. The curves show the energy dependence predicted by QCD, using $\alpha_{s}\left(m_{\tau}\right)$ as input. The corresponding extrapolated $\alpha_{s}\left(M_{Z}\right)$ values are shown at the bottom, where the shaded band displays the $\tau$ decay result within errors [44].

the fundamental QCD coupling [31,32]. The experimental measurement $R_{\tau, V+A}=3.478 \pm 0.011$ implies [44]

$\alpha_{s}\left(m_{\tau}\right)=0.344 \pm 0.005_{\exp } \pm 0.007_{\mathrm{th}}$.

The strong coupling measured at the $\tau$ mass scale is significantly larger than the values obtained at higher energies. From the hadronic decays of the $Z$, one gets $\alpha_{s}\left(M_{Z}\right)=0.1191 \pm 0.0027$ [16], which differs from $\alpha_{s}\left(m_{\tau}\right)$ by more than $20 \sigma$. After evolution up to the scale $M_{Z}$ [45], the strong coupling constant in (4) decreases to [44]

$\alpha_{s}\left(M_{Z}\right)=0.1212 \pm 0.0011$,

in excellent agreement with the direct measurements at the $Z$ peak and with a better accuracy. The comparison of these two determinations of $\alpha_{s}$ in two very different energy regimes, $m_{\tau}$ and $M_{Z}$, provides a beautiful test of the predicted running of the QCD coupling; i.e., a very significant experimental verification of asymptotic freedom. 


\section{5. $\left|V_{u s}\right|$ determination from tau decays}

The separate measurement of the $|\Delta S|=0$ and $|\Delta S|=1$ tau decay widths provides a very clean determination of $V_{u s}[46,47]$. To a first approximation the Cabibbo mixing can be directly obtained from experimental measurements, without any theoretical input. Neglecting the small $\mathrm{SU}(3)$-breaking corrections from the $m_{s}-m_{d}$ quark-mass difference, one gets:

$\left|V_{u s}\right|^{\mathrm{SU}(3)}=\left|V_{u d}\right|\left(\frac{R_{\tau, S}}{R_{\tau, V+A}}\right)^{1 / 2}=0.210 \pm 0.003$.

We have used $\left|V_{u d}\right|=0.97377 \pm 0.00027$ [6], $R_{\tau}=3.640 \pm 0.010$ and the value $R_{\tau, S}=$ $0.1617 \pm 0.0040$ [47], which results from the most recent BaBar [48] and Belle [49] measurements of Cabibbo-suppressed tau decays [50]. The new branching ratios measured by BaBar and Belle are all smaller than the previous world averages, which translates into a smaller value of $R_{S}$ and $\left|V_{u s}\right|$. For comparison, the previous value $R_{\tau, S}=0.1686 \pm 0.0047$ [44] resulted in $\left|V_{u s}\right|^{\mathrm{SU}(3)}=0.215 \pm 0.003$.

This rather remarkable determination is only slightly shifted by the small SU(3)-breaking contributions induced by the strange quark mass. These corrections can be estimated through a QCD analysis of the differences $[46,47,51-58]$

$\delta R_{\tau}^{k l} \equiv \frac{R_{\tau, V+A}^{k l}}{\left|V_{u d}\right|^{2}}-\frac{R_{\tau, S}^{k l}}{\left|V_{u s}\right|^{2}}$.

The only non-zero contributions are proportional to the mass-squared difference $m_{s}^{2}-m_{d}^{2}$ or to vacuum expectation values of $\mathrm{SU}(3)$-breaking operators such as $\delta O_{4} \equiv\left\langle 0\left|m_{s} \bar{s} s-m_{d} \bar{d} d\right| 0\right\rangle \approx$ $(-1.4 \pm 0.4) \cdot 10^{-3} \mathrm{GeV}^{4}[46,51]$. The dimensions of these operators are compensated by corresponding powers of $m_{\tau}^{2}$, which implies a strong suppression of $\delta R_{\tau}^{k l}$ [51]:

$$
\begin{aligned}
\delta R_{\tau}^{k l} \approx 24 S_{\mathrm{EW}} & \left\{\frac{m_{s}^{2}\left(m_{\tau}\right)}{m_{\tau}^{2}}\left(1-\epsilon_{d}^{2}\right) \Delta_{k l}\left(\alpha_{s}\right)\right. \\
& \left.-2 \pi^{2} \frac{\delta O_{4}}{m_{\tau}^{4}} Q_{k l}\left(\alpha_{s}\right)\right\},
\end{aligned}
$$

where $\epsilon_{d} \equiv m_{d} / m_{s}=0.053 \pm 0.002$ [59]. The perturbative corrections $\Delta_{k l}\left(\alpha_{s}\right)$ and $Q_{k l}\left(\alpha_{s}\right)$ are known to $O\left(\alpha_{s}^{3}\right)$ and $O\left(\alpha_{s}^{2}\right)$, respectively $[51,58]$.
The theoretical analysis of $\delta R_{\tau} \equiv \delta R_{\tau}^{00}$ involves the two-point vector and axial-vector correlators, which have transverse $(J=1)$ and longitudinal $(J=0)$ components. The longitudinal contribution to $\Delta_{00}\left(\alpha_{s}\right)$ shows a rather pathological behaviour, with clear signs of being a non-convergent perturbative series. Fortunately, the corresponding longitudinal contribution to $\delta R_{\tau}$ can be estimated phenomenologically with a much better accuracy, $\left.\delta R_{\tau}\right|^{L}=0.1544 \pm 0.0037$ $[46,60]$, because it is dominated by far by the well-known $\tau \rightarrow \nu_{\tau} \pi$ and $\tau \rightarrow \nu_{\tau} K$ contributions. To estimate the remaining transverse component, one needs an input value for the strange quark mass. Taking the range $m_{s}\left(m_{\tau}\right)=(100 \pm 10) \mathrm{MeV}$ $\left[m_{s}(2 \mathrm{GeV})=(96 \pm 10) \mathrm{MeV}\right]$, which includes the most recent determinations of $m_{s}$ from QCD sum rules and lattice QCD [60], one gets finally $\delta R_{\tau, t h}=0.216 \pm 0.016$, which implies [47]

$$
\begin{aligned}
\left|V_{u s}\right| & =\left(\frac{R_{\tau, S}}{\frac{R_{\tau, V+A}}{\left|V_{u d}\right|^{2}}-\delta R_{\tau, \mathrm{th}}}\right)^{1 / 2} \\
& =0.2165 \pm 0.0026_{\exp } \pm 0.0005_{\mathrm{th}} .
\end{aligned}
$$

A larger central value $\left(\left|V_{u s}\right|=0.2212 \pm 0.0031\right)$ is obtained with the old world average for $R_{\tau, S}$.

Sizeable changes on the experimental determination of $R_{\tau, S}$ are to be expected from the full analysis of the huge BaBar and Belle data samples. In particular, the high-multiplicity decay modes are not well known at present. Thus, the result (8) could easily fluctuate in the near future. However, it is important to realize that the final error of the $V_{u s}$ determination from $\tau$ decay is completely dominated by the experimental uncertainties. If $R_{\tau, S}$ is measured with a $1 \%$ precision, the resulting $V_{u s}$ uncertainty will get reduced to around $0.6 \%$, i.e. \pm 0.0013 , making $\tau$ decay the best source of information about $V_{u s}$.

An accurate measurement of the invariantmass distribution of the final hadrons could make possible a simultaneous determination of $V_{u s}$ and the strange quark mass, through a correlated analysis of several weighted differences $\delta R_{\tau}^{k l}$. The extraction of $m_{s}$ suffers from theoretical uncertainties related to the convergence of the perturbative series $\Delta_{k l}^{L+T}\left(\alpha_{s}\right)$, which makes necessary a better understanding of these corrections. 


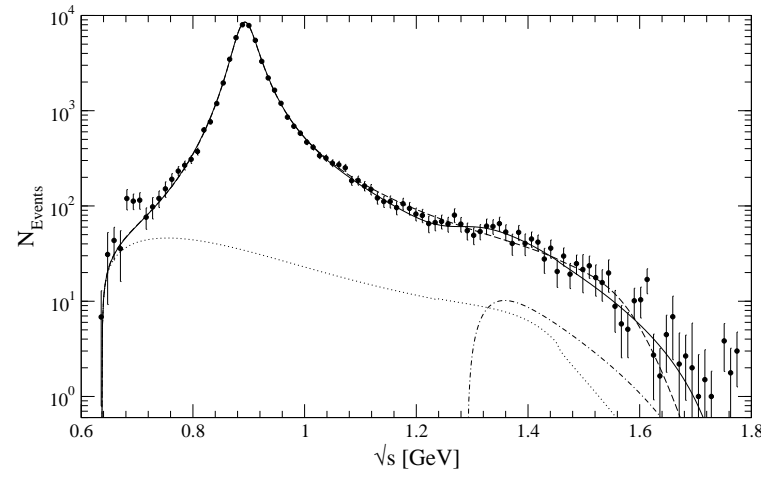

Figure 2. Theoretical description [61] (solid line) of the Belle $\tau^{-} \rightarrow \nu_{\tau} K_{S} \pi^{-}$data [62]. The $K^{*^{\prime}}$ (dashed-dotted) and scalar (dotted) contributions are also shown.

\section{6. $\tau \rightarrow \nu_{\tau} K \pi$ and $K \rightarrow \pi l \bar{\nu}_{l}$}

The decays $\tau \rightarrow \nu_{\tau} K \pi$ probe the same hadronic form factors investigated in $K_{l 3}$ processes, but they are sensitive to a much broader range of invariant masses. A theoretical understanding of the form factors can be achieved, using analyticity, unitarity and some general properties of QCD, such as chiral symmetry and the shortdistance asymptotic behaviour. Figure 2 compares the resulting theoretical description of the $\tau$ decay spectrum [61] with the recent Belle measurement [62]. At low values of $s$ there is clear evidence of the scalar contribution, which was predicted using a careful analysis of $K \pi$ scattering data $[60,63]$. From the measured $\tau$ spectrum one obtains $M_{K^{*}}=895.3 \pm 0.2 \mathrm{MeV}$ and $\Gamma_{K^{*}}=47.5 \pm 0.4 \mathrm{MeV}[61]$. Since the absolute normalization is fixed by $K_{l 3}$ data to be $\left|V_{u s}\right| f_{+}^{K^{0} \pi^{-}}(0)=0.21664 \pm 0.00048$ [13], one gets then a theoretical prediction for the branching fraction, $\operatorname{Br}\left(\tau^{-} \rightarrow \nu_{\tau} K_{S} \pi^{-}\right)=0.427 \pm 0.024 \%$, in good agreement with the Belle measurement $0.404 \pm 0.013 \%$, although slightly larger.

The $\tau$ determination of the vector form factor $f_{+}^{K \pi}(s)$ provides precise values for its slope and curvature [61], $\lambda_{+}^{\prime}=(25.2 \pm 0.3) \cdot 10^{-3}$ and $\lambda_{+}^{\prime \prime}=$ $(12.9 \pm 0.3) \cdot 10^{-4}$, in agreement but more precise than the corresponding $K_{l 3}$ measurements [13].

\section{Acknowledgements}

I want to thank the hospitality of the CERN Theory Division. This work has been supported by MEC, Spain (grants FPA2007-60323 and Consolider-Ingenio 2010 CSD2007-00042, CPAN) and by the EU Contract MRTN-CT-2006-035482 (FLAVIAnet).

\section{REFERENCES}

1. A. Pich, The Standard Model of Electroweak Interactions, in Proc. 2006 European School of High-Energy Physics (Aronsborg, Sweden), ed. R. Fleischer, CERN-2007-005 p. 1, arXiv:0705.4264 [hep-ph].

2. A. Pich, Int. J. Mod. Phys. A 21 (2006) 5652; Nucl. Phys. B (Proc. Suppl.) 169 (2007) 393.

3. A. Lusiani, Nucl. Phys. B (Proc. Suppl.) 144 (2005) 105.

4. MuLan Collaboration, Phys. Rev. Lett. 99 (2007) 032001.

5. FAST Collaboration, Phys. Lett. B 663 (2008) 172.

6. W.-M. Yao et al., Review of Particle Physics, J. Phys. G 33 (2006) 1 and 2007 partial update for edition 2008 http://pdg.lbl.gov/.

7. Belle Collaboration, Phys. Rev. Lett. 99 (2007) 011801.

8. V.V. Anashin et al., JETP Lett. 85 (2007) 347.

9. X.H. Mo, Nucl. Phys. B (Proc. Suppl.) 169 (2007) 132.

10. P. Ruiz-Femenía and A. Pich, Phys. Rev. D 64 (2001) 053001; Nucl. Phys. B (Proc. Suppl.) 121 (2003) 205.

11. M.B. Voloshin, Phys. Lett. B 556 (2003) 153.

12. B.H. Smith and M.B. Voloshin, Phys. Lett. B 324 (1994) 117; 333 (1994) 564.

13. FlaviaNet Working Group on Kaon Decays, arXiv:0801.1817 [hep-ph].

14. V. Cirigliano and I. Rosell, Phys. Rev. Lett. 99 (2007) 231801; JHEP 0710 (2007) 005.

15. V. Cirigliano et al., Eur. Phys. J. C 23 (2002) 121.

16. The LEP Collaborations ALEPH, DELPHI, L3 and OPAL and the LEP Electroweak Working Group, arXiv:0712.0929 [hep-ex]; 
http://www.cern.ch/LEPEWWG/.

17. V. Cirigliano, G. Isidori and V. Porretti, Nucl. Phys. B 763 (2007) 2287.

18. B. Grinstein et al., Nucl. Phys. B 763 (2007) 35; 728 (2005) 121.

19. S. Antusch et al., JHEP 0611 (2006) 090.

20. E. Arganda and M.J. Herrero, Phys. Rev. D 73 (2006) 055003. E. Arganda, M.J. Herrero and J. Portolés, arXiv:0803.2039 [hep-ph].

21. M. Blanke et al., JHEP 0705 (2007) 013.

22. M. Ciuchini et al., Nucl. Phys. B 783 (2007) 112.

23. P. Paradisi, JHEP 0602 (2006) 050; 0510 (2005) 006.

24. BaBar Collaboration, Phys. Rev. Lett. 100 (2008) 071802; 99 (2007) 251803; 98 (2007) 061803; 96 (2006) 041801; 95 (2005) 191801, 041802; 92 (2004) 121801.

25. Belle Collaboration, arXiv:0705.0650 [hepex]; Phys. Lett. B 664 (2008) 35; 660 (2008) $154 ; 648$ (2007) 341; 640 (2006) 138; 639 (2006) 159; 632 (2006) 51; 622 (2005) 218; 589 (2004) 103; Phys. Rev. Lett. 93 (2004) 081803; 92 (2004) 171802.

26. M. Bona et al., arXiv:0709.0451 [hep-ex].

27. T. Mori, Nucl. Phys. B (Proc. Suppl.) 169 (2007) 166.

28. J-PARC P21-LoI (2006).

29. J-PARC P20-LoI (2006).

30. E. Braaten, Phys. Rev. Lett. 60 (1988) 1606; Phys. Rev. D 39 (1989) 1458.

31. S. Narison and A. Pich, Phys. Lett. B 211 (1988) 183.

32. E. Braaten, S. Narison and A. Pich, Nucl. Phys. B 373 (1992) 581.

33. F. Le Diberder and A. Pich, Phys. Lett. B 286 (1992) 147.

34. A. Pich, Nucl. Phys. B (Proc. Suppl.) 39B,C (1995) 326.

35. W.J. Marciano and A. Sirlin, Phys. Rev. Lett. 61 (1988) 1815.

36. E. Braaten and C.S. Li, Phys. Rev. D 42 (1990) 3888.

37. J. Erler, Rev. Mex. Phys. 50 (2004) 200.

38. P.A. Baikov, K.G. Chetyrkin and J.H. Kühn, arXiv:0801.1821 [hep-ph].

39. A.A. Pivovarov, Z. Phys. C 53 (1992) 461.

40. F. Le Diberder and A. Pich, Phys. Lett. B
289 (1992) 165.

41. ALEPH Collaboration, Phys. Rep. 421 (2005) 191; Eur. Phys. J. C 4 (1998) 409; Phys. Lett. B 307 (1993) 209.

42. CLEO Collaboration, Phys. Lett. B 356 (1995) 580.

43. OPAL Collaboration, Eur. Phys. J. C 7 (1999) 571.

44. M. Davier et al., Rev. Mod. Phys. 78 (2006) 1043; arXiv:0803.0979 [hep-ph].

45. G. Rodrigo, A. Pich and A. Santamaria, Phys. Lett. B 424 (1998) 367.

46. E. Gámiz et al., Phys. Rev. Lett. 94 (2005) 011803; JHEP 0301 (2003) 060.

47. E. Gámiz et al., PoS KAON 008 (2007).

48. BaBar Collaboration, Phys. Rev. Lett. 100 (2008) 011801; Phys. Rev. D 76 (2007) 051104 .

49. Belle Collaboration, Phys. Lett. B 654 (2007) 65; 643 (2006) 5.

50. S. Banerjee, PoS KAON 009 (2007).

51. A. Pich and J. Prades, JHEP 9910 (1999) 004; 9806 (1998) 013.

52. S. Chen et al., Eur. Phys. J. C 22 (2001) 31. M. Davier et al., Nucl. Phys. B (Proc. Suppl.) 98 (2001) 319.

53. K.G. Chetyrkin, J.H. Kühn and A.A. Pivovarov, Nucl. Phys. B 533 (1998) 473.

54. J.G. Körner, F. Krajewski and A.A. Pivovarov, Eur. Phys. J. C 20 (2001) 259.

55. K. Maltman and C.E. Wolfe, Phys. Lett. B 639 (2006) 283.

56. J. Kambor and K. Maltman, Phys. Rev. D 62 (2000) 093023; 64 (2001) 093014.

57. K. Maltman, Phys. Rev. D 58 (1998) 093015.

58. P.A. Baikov, K.G. Chetyrkin and J.H. Kühn, Phys. Rev. Lett. 95 (2005) 012003.

59. H. Leutwyler, Phys. Lett. B 378 (1996) 313.

60. M. Jamin, J.A. Oller and A. Pich, Phys. Rev. D 74 (2006) 074009.

61. M. Jamin, A. Pich and J. Portolés, Phys. Lett. B 640 (2006) 176; 664 (2008) 78.

62. Belle Collaboration, Phys. Lett. B 654 (2007) 65.

63. M. Jamin, J.A. Oller, and A. Pich, Nucl. Phys. B 587 (2000) 331; 622 (2002) 279; Eur. Phys. J. C 24 (2002) 237. 